\title{
Sustained release biodegradable solid lipid microparticles: Formulation, evaluation and statistical optimization by response surface methodology
}

\author{
MUHAMMAD HANIF ${ }^{1}$ \\ HAFEEZ ULLAH KHAN ${ }^{1,2, *}$ \\ SAMINA AFZAL ${ }^{1}$ \\ ASIF MAHMOOD 3 \\ SAFIRAH MAHEEN ${ }^{2}$ \\ KHURRAM AFZAL ${ }^{4}$ \\ NABILA IQBAL ${ }^{2}$ \\ MEHWISH ANDLEEB ${ }^{5}$ \\ NAZAR ABBAS 6,7 \\ ${ }^{1}$ Faculty of Pharmacy, Bahauddin \\ Zakariya University, Multan, Pakistan \\ ${ }^{2}$ Faculty of Pharmacy, University \\ of Sargodha, Sargodha, Pakistan \\ ${ }^{3}$ Institute of Pharmacy \\ Physiology and Pharmacology \\ University of Agriculture Faisalabad \\ ${ }^{4}$ Department of Food sciences \\ Bahauddin Zakariya University \\ Multan, Pakistan \\ ${ }^{5}$ Faculty of Pharmacy and Alternative \\ Medicines, Islamia University \\ Bahawalpur, Pakistan \\ ${ }^{6}$ Research and Development \\ Mass Pharma(Pvt) Ltd, Lahore, Pakistan \\ ${ }^{7}$ Rashid Latif College of Pharmacy \\ Lahore, Pakistan
}

Accepted July 18, 2017

Published online September 12, 2017

\begin{abstract}
For preparing nebivolol loaded solid lipid microparticles (SLMs) by the solvent evaporation microencapsulation process from carnauba wax and glyceryl monostearate, central composite design was used to study the impact of independent variables on yield $\left(Y_{1}\right)$, entrapment efficiency $\left(Y_{2}\right)$ and drug release $\left(Y_{3}\right)$. SLMs having a 10-40 $\mu \mathrm{m}$ size range, with good rheological behavior and spherical smooth surfaces, were produced. Fourier transform infrared spectroscopy, differential scanning calorimetry and X-ray diffractometry pointed to compatibility between formulation components and the zeta-potential study confirmed better stability due to the presence of negative charge $(-20$ to $-40 \mathrm{mV})$. The obtained outcomes for $Y_{1}(29-86 \%), Y_{2}(45-83 \%)$ and $Y_{3}(49-86 \%)$ were analyzed by polynomial equations and the suggested quadratic model were validated. Nebivolol release from SLMs at $\mathrm{pH} 1.2$ and 6.8 was significantly $(p<0.05)$ affected by lipid concentration. The release mechanism followed Higuchi and zero order models, while $n>0.85$ value (Korsmeyer-Peppas) suggested slow erosion along with diffusion. The optimized SLMs have the potential to improve nebivolol oral bioavailability.
\end{abstract}

Keywords: central composite design, differential scanning calorimetry, solid lipid microparticles, microencapsulation, nebivolol, carnauba wax

Cardiovascular diseases such as hypertension cause millions of deaths every year and hypertension may further cause congestive heart failure, myocardial infarction, stroke and renal diseases (1). Lifestyle modifications alone cannot manage hypertension and patients

\footnotetext{
*Correspondence; e-mail: qarani_pharmacist@yahoo.com
} 
have to rely on pharmacological intervention. The existing conventional drug delivery systems for such diseases cannot yield optimal therapeutic responses and fluctuation or dose missing may occur. This situation surely demands sustained drug delivery for achieving increased heart rate control (2).

Today, most pharmaceutical scientists are engaged in formulating an ideal oral drug delivery system that could offer controlled drug release to achieve the desired therapeutic outcome in an appreciably improved way (2). Oral sustained release formulations have been gaining ample consideration over the last three decades because of their significant therapeutic benefits such as flexibility in formulation, ease of administration and particularly enhanced patient compliance. Microspheres, particularly based on natural biodegradable lipids (solid lipid microparticles) have attracted significant attention for offering sustained drug delivery (3-5).

SLMs exhibit enhanced physical stability, safety, biocompatibility; they improve drug aqueous solubility and provide higher plasma drug availability with improved membrane permeability (4-5). Moreover, they are characterized by dual loading ability (i.e. lipophilic and hydrophilic drugs) and are suitable and economical for large scale production (2). SLMs can prolong residence time at the absorption site (2) and can be formulated from solid lipids such as carnauba wax (CW) and glyceryl monostearate (GMS) because they exhibit excellent biocompatibility, biodegradability (4), lack of toxicity and ease of production (4-6). CW and GMS SLMs can make drug release sustained and enhance drug bioavailability (2-3). SLMs offer effective drug release control for a prolonged time period, causing a significant decrease in the number of doses and associated side effects, particularly in patients with chronic diseases (5). Definitely, such chronic diseases like hypertension demand a sustained/controlled release drug delivery of a therapeutic agent to obtain better heart rate control and utilization of SLMs is found to be one of the most promising strategies among numerous available strategies for raising therapeutic compliance (3).

For the management of hypertension and to attain optimal therapeutic responses, $\beta$-blockers are considered a better option than other antihypertensive drugs, like calcium channel blockers, which cause rebound hypertension and cough and are considered as first line antihypertensive drugs (6). Compatibility of $\beta$-blockers with lipid excipients has been established (7) and some $\beta$-blockers such as propranolol (8), atenolol, metoprolol (89), pindolol, labetolol (7) and carvedilol (9) have been encapsulated in solid lipid based polymers for oral $(8,10)$, topical $(11)$, intranasal (12) and ophthalmic (13) routes of administration. $\beta$-blockers have also been formulated in the form of mucoadhesive tablets containing propranolol loaded chitosan-gelatin microparticles (14-15).

Nebivolol (NEB) is a new third-generation highly selective $\beta$-blocker that causes vasodilatation through nitric oxide (NO) release and it is employed for the first-line management of hypertension. NEB is highly effective and acceptable compared to other antihypertensive agents but it demands frequent dosing due to its bioavailability $(<40 \%)$ and plasma half-life $(2 \mathrm{~h}$ ). Low NEB oral bioavailability is a consequence of its poor solubility $(6,16)$. Hence, it demands improvement of solubility, dissolution and bioavailability (3) and the use of SLMs in this situation seems to be a better strategy compared to polymeric particles because SLMs can overcome the drawbacks like inefficient biodegradation, polymer accumulation effects and toxicity associated with polymeric microparticles (5).

The aim of the present study was to enhance NEB bioavailability by formulating sustained release NEB-loaded SLMs using the solvent evaporation microencapsulation tech- 
nique. CW and GMS were used as release retarding materials because these lipophilic biomaterials are biodegradable and biocompatible, which makes them ideal candidates for this purpose. The effect of formulation factors on physicochemical and biopharmaceutical properties of SLMs was statistically analyzed with the aid of a central composite rotatable design (CCRD), response surface methodology, and numerical optimization techniques were applied to obtain a formulation with fine-tuned properties.

\section{EXPERIMENTAL}

\section{Materials}

NEB was a generous gift of NabiQasim Pharmaceuticals (Pvt) Ltd, Lahore, Pakistan. Potassium dihydrogen phosphate and Tween-20 (T-20) were purchased from Merck, Germany. CW, GMS and the cellulose dialysis tube were purchased form Sigma-Aldrich, USA, while potassium bromide of IR grade was obtained from Fischer Scientific, UK. All chemicals and reagents used were of analytical grade.

\section{Experimental design (CCRD)}

Different factors such as concentration, type of lipid, microencapsulation technique, concentration and type of surfactant, stirring speed and stirring time affect the yield and features of SLMs. Conventional experimentation for optimization needs variation of one variable while keeping all others at a constant level, which may lack elaboration of interactions of different variables at the same time and also cause excessive consumption of time and excipients. The effect of different controlled independent factors on different responses/features of a formulation can be studied effectively with the help of Central composite Rotatable Design (CCRD), which is a statistical procedure for optimization of formulations and is usually employed using software like Design Expert (version 8.0.6.1 Stat-ease, Inc.). CCRD also suggests a model equation for a specific response as a function of various controlled independent variables (17-18).

In this study, formulation factors investigated for their impact on various responses of SLMs were CW concentration $\left(X_{1}\right)$, GMS concentration $\left(X_{2}\right)$ and T-20 concentration $\left(X_{3}\right)$, as shown in Table I. Dependent variables (responses) for this study include percentage yield $\left(Y_{1}\right)$, entrapment efficiency $\left(Y_{2}\right)$ and drug release at $12 \mathrm{~h}\left(Y_{3}\right)$. Statistical software (Design Expert) generated representative combinations of these three factors at five levels and the entire design consisted of 20 runs of experiments (15) with factorial (8), axial (6) and central (6) points (Table I). Some preliminary trials were performed in order to identify appropriate levels of all investigated factors (independent variables) before applying the design.

\section{Preparation of SLMs (solvent evaporation method)}

NEB-loaded SLMs were prepared by the solvent evaporation method. GMS and CW were dissolved in $50 \mathrm{~mL}$ of chloroform and then NEB was dissolved in this solution of solid lipids. The prepared lipid solution was added to a premade hot aqueous solution of T-20, maintained at $75^{\circ} \mathrm{C}$ and homogenized at $3000 \mathrm{rpm}$ for 2 hours. The resulting pre-emulsion 


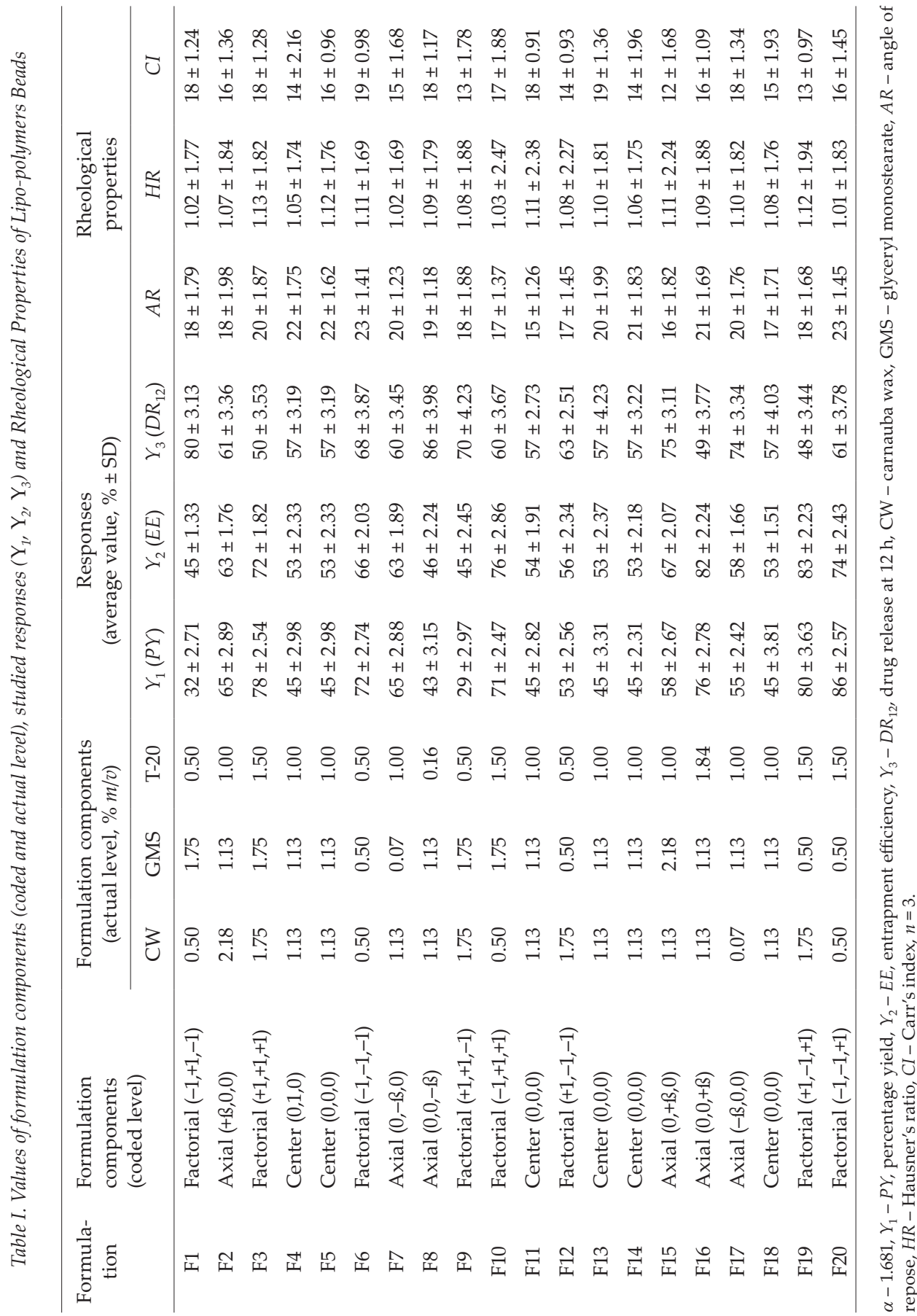


was poured into cold water $\left(1-4^{\circ} \mathrm{C}\right)$ and stirred with a magnetic stirrer. SLMs were allowed to recrystallize at room temperature, filtered using a $0.45 \mu \mathrm{m}$ filter paper and dried using a desiccator. Every formulation was prepared three times and the same procedure was repeated to yield all formulations by varying three variables, as shown in Table I. The aqueous phase volume $(250 \mathrm{~mL})$ and the amount of the drug $(10 \mathrm{mg})$ were kept constant $(19)$.

\section{Characterization of SLMs}

Rheological studies. - Rheological studies concern evaluation of flow properties of SLM formulations. Appropriate flow behavior of SLMs is mandatory if SLMs are to be converted into tablets, capsules or some other dosage form. These studies included evaluation of bulk density, tapped density, Carr's compressibility index (CI), Hausner's ratio (HI) and angle of repose $(A R)$.

Bulk density. - For bulk density $\left(\rho_{\mathrm{b}}\right)$ calculation, the bulk volume $\left(V_{\mathrm{b}}\right)$ occupied by a pre-weighed amount of SLMs $(m)$ was observed from a graduated cylinder. Bulk density was calculated with the help of the following formula $(4,19)$ :

$$
\rho_{\mathrm{b}}=\frac{m}{V_{\mathrm{b}}}
$$

where $V_{\mathrm{b}}$ is the bulk volume and $m$ is the mass of SLMs.

Tapped density. - For tapped density measurement, a graduated cylinder having a preweighed amount of SLMs was tapped for a specified number of tapings so that SLMs attained a plateau condition. The tapped volume $\left(V_{\mathrm{t}}\right)$ occupied by SLMs after tapping was observed $(4,19)$. Calculation of tapped density $\left(\rho_{t}\right)$ was performed using the of following formula;

$$
\rho_{\mathrm{t}}=\frac{m}{V_{\mathrm{t}}}
$$

where $V_{\mathrm{t}}$ is the tapped volume of SLMs.

Carr's compressibility index. - The compressibility index (CI) of SLMs is also an indicator of flow behavior and was determined with the following formula (19).

$$
C I=\frac{V_{\mathrm{b}}-V_{\mathrm{t}}}{V_{\mathrm{t}}} \times 100
$$

The value of Carr's index from 12-19\% indicates a good flow character, while its value higher than $21 \%$ suggests poor flow properties.

Hausner's ratio. - Hausner's ratio is the ratio of tapped density to bulk density of a material. It was also applied to calculate the flow behavior of SLMs.

$$
H R=\frac{\rho_{\mathrm{t}}}{\rho_{\mathrm{b}}}
$$

where $\rho_{\mathrm{t}}$ is the tapped density and $\rho_{\mathrm{b}}$ represents the bulk density of SLMs. The ratio higher than 1.25 shows poor flow behavior and its value lower than 1.25 indicates good flow properties (19). 
Angle of repose. - A specified amount of SLMs was passed through a funnel on a plain sheet of paper. The falling SLMs made a heap on the sheet of paper. The height $(h)$ and radius $(r)$ of the heap were measured and these values were used to determine the angle of repose with the help of the following formula:

$$
\tan \theta=h / r
$$

Free flow behavior of SLMs is confirmed if the angle of repose value is less than $30^{\circ}$ (19).

\section{Percentage yield}

To calculate percentage yield (PY), a mass of finally prepared and dried SLMs was taken and the obtained mass was divided by the total amount of all solid lipid components used to prepare SLMs (20).

\section{Entrapment efficiency and drug loading}

The amount of NEB entrapped in SLMs was also calculated. For this purpose, a specified amount of SLMs was crushed in a mortar and then dispersed in phosphate buffer ( $\mathrm{pH}$ 7.4) for $24 \mathrm{~h}$ under continuous stirring. The solution was diluted up to 5 times by the use of phosphate buffer ( $\mathrm{pH} 7.4$ ) after filtration. Absorbance was measured at $286 \mathrm{~nm}$ using a UV-visible spectrophotometer (Pharma Spec 1700 Shimadzu, Japan). Calculations of the drug amount encapsulated $(E E)$ in SLMs and drug loading $(D L)$ were performed using the following formulas (20).

$$
\begin{gathered}
E E=(\text { Actual drug amount } / \text { Initial drug amount added in formulation }) \times 100 \\
\qquad D L=(\text { Quantity of the drug in SLMs } / \text { mass of SLMs }) \times 100
\end{gathered}
$$

In vitro drug release

In vitro drug release studies of all formulations were executed with the aid of a USP type-II apparatus (PT-DT7, Pharma Test Germany) in $0.1 \mathrm{~mol} \mathrm{~L}^{-1} \mathrm{HCl}(\mathrm{pH} 1.2)$ and in phosphate buffer ( $\mathrm{pH}$ 6.8) as dissolution media at $37 \pm 0.5^{\circ} \mathrm{C}$ at a rotation speed of $50 \mathrm{rpm}$. For every formulation, including the optimized ones, a sample of SLMs equivalent to $5 \mathrm{mg}$ of NEB was placed into a cellulose dialysis tube containing $5 \mathrm{~mL}$ of dissolution medium. The dialysis tube was then attached to a paddle to maintain the sink condition in the dissolution vessel containing $900 \mathrm{~mL}$ of dissolution medium. After a specified time interval, a 5 $\mathrm{mL}$ sample was withdrawn from each dissolution vessel and an equal volume $(5 \mathrm{~mL})$ of freshly prepared and pre-warmed $\left(37^{\circ} \mathrm{C}\right)$ dissolution medium was added into the same vessel. The obtained dissolution samples were diluted up to 100 times with distilled water and absorbance of the diluted sample was measured at $286 \mathrm{~nm}$ to determine the content of NEB in SLMs $(2,20)$.The study was carried out for $2 \mathrm{~h}$ at $\mathrm{pH} 1.2$ and for $12 \mathrm{~h}$ at $\mathrm{pH} 6.8$. The drug release study included all the designed formulations and one optimized formulation in triplicate. 


\section{Drug release data analysis}

To study the drug release mechanism, the drug release data of all formulations were further analyzed with the help of different kinetics models such as zero order, first order, Korsmeyer-Peppas, Hixson-Crowell and Higuchi's models as follows (2, 20):

$$
\begin{gathered}
F_{\mathrm{t}}=K_{0} t \\
\log F=\log F_{0}-K_{\mathrm{t}} / 2 \cdot 303 \\
F=K_{\mathrm{H}} t^{1 / 2} \\
F_{0}^{1 / 3}-F_{\mathrm{t}}^{1 / 3}=K_{H C} \times t \\
\frac{M_{\mathrm{t}}}{M_{\infty}}=K_{3} t^{n}
\end{gathered}
$$

Zero and first order rate constants are denoted $K_{0}$ and $K_{1}$, respectively. Similarly, $K_{\mathrm{H}}$ and $K_{\mathrm{HC}}$ represent the Higuchi and Hixson-Crowell rate constants. Drug concentration at time $t$ was denoted $F / F_{\mathrm{t}}$, and the initial drug concentration was marked $F_{0} . K_{3}$ denotes the Korsmeyer-Peppas rate constant while $n$ represents an exponent of the drug release mechanism. The value of $n$ lower than 0.43 shows the Fickian mechanism while the value of $n$ higher than 0.43 and lower than 0.85 indicates the non-Fickian drug release mechanism. The value of $n$ higher than 0.85 suggests slow erosion plus a diffusion drug release mechanism (20-21).

\section{Fourier transform infrared spectroscopy (FTIR)}

Compatibility of NEB with CW and GMS was analyzed with the help of an FTIR spectrophotometer (IR Prestage 21 Shimadzu). The FTIR spectra of the drug, CW, GMS and the drug loaded optimized formulation were recorded using $\mathrm{KBr}$ pellets, with the scanning range of 4000 to $400 \mathrm{~cm}^{-1}$, at a $2 \mathrm{~cm}^{-1}$ resolution. Hydraulic pressure of $150 \mathrm{~kg} \mathrm{~cm}^{-2}$ was used in the study (4-5).

\section{Differential scanning calorimetry (DSC)}

Drug-polymer interaction can be also performed with the help of a Thermal analyzer (SDT Q600 TA USA). The DSC analysis of NEB, CW, GMS and NEB-loaded optimized formulation was performed on an SDT-Q600 TA USA to examine any possible drug-lipid matrix interaction. CW, GMS, NEB and NEB-loaded SLMs were finely triturated and the prepared samples were then heated at a heating rate of $10{ }^{\circ} \mathrm{C} / \mathrm{min}$ from 0 to $240{ }^{\circ} \mathrm{C}$ in a

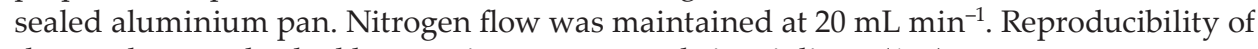
the results was checked by running every sample in triplicate (4-5).

\section{X-ray powder diffraction studies}

X-ray diffraction studies were conducted with the intention to observe the impact of the microencapsulation process on NEB crystallinity (5). The samples were subjected to 
irradiation with monochromatized X-rays of $\mathrm{Cu}-\mathrm{K} \alpha$ having a voltage of $40 \mathrm{kV}$ using a D-8 advance X-ray diffractometer (Bruker AXS, USA) at a $40 \mathrm{~mA}$ current. Scanning of samples such as NEB alone, CW alone, GMS alone and of the optimized formulation of drug loaded

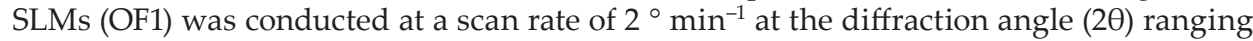
from 0 to $45^{\circ}(4-5,21)$.

\section{Scanning electron microscopy (SEM)}

The shape and surface morphology of the optimized SLMs formulation and the formulation showing maximum drug release at $12 \mathrm{~h}$ were examined using a scanning electron microscope (JSM-840, Joel Instruments, Tokyo, Japan). The SLM sample was positioned on a double adhesive tape in the form of drops and the adhesive tape was then stuck to an aluminum stub. After complete evaporation from this prepared sample, gold coating of stubs was conducted to make them electrically conductive under an argon atmosphere. Photomicrographs of SLMs were obtained at 500x magnification at $10 \mathrm{kV}(4-5)$.

\section{Zeta-potential and particle size measurements}

Measurement of the charge over the surface of finely prepared NEB-loaded optimized SLMs was performed by evaluating their electrophoretic mobility in a U-shaped tube at 25 ${ }^{\circ} \mathrm{C}$ using a Malvern Zetasizer (Zetasizer Ver System; Malvern Instruments Ltd., UK). Average size and size distribution of drug loaded SLMs were also determined and for this purpose, SLMs were added in a cuvette containing deionized water, which was then placed in the Zetasizer for size measurement $(4-5,21)$.

\section{Data analysis and validation}

With the aid of ANOVA provided in the Design-Expert software, each suggested polynomial equation and model for each response was validated statistically. The suggested model for each response was further analyzed on the basis of values of the predicted residual sum of squares (PRESS), multiple correlation coefficient $\left(R^{2}\right)$, adjusted multiple correlation coefficient (adjusted $R^{2}$ ), and coefficient of variation $(\mathrm{CV})$. The level of significance was set at 0.05 . Two dimensional contour and 3D surface plots were plotted to assess the effects of independent variables on dependent variables (17-18). Comparison was also made between experimental and predicted values. The responses followed the quadratic model and the suggested equation is given below:

$$
Y=b_{0}+b_{1} X_{1}+b_{2} X_{2}+b_{3} X_{3}+b_{12} X_{1} X_{2}+b_{13} X_{1} X_{3}+b_{23} X_{2} X_{3}+b_{11} X_{1}^{2}+b_{22} X_{2}^{2}+b_{33} X_{3}^{2}
$$

where $Y$ denotes the studied dependent response. Intercept $b_{0}$ represents the average of all outcomes from 20 runs while regression coefficients $\left(b_{1}\right.$ to $\left.b_{33}\right)$ were the calculated average values of response $Y$ by changing one factor alone and changing two factors at the same time. Coded levels of independent factors were denoted $X_{1}, X_{2}$ and $X_{3}$, indicating average values of the response obtained from changing one factor from the low to high level. Interaction terms for a combination of factors were denoted $X_{1} X_{2}, X_{2} X_{3}, X_{1} X_{3}$ and they indicated the average change in response that occurred due to the change of two factors at the same time (21). A negative or a positive sign showed the antagonistic or synergetic impact 
of the factor/factors on the response. To get an optimized formulation, help was also sought of the desirability approach, numerical and graphical optimization tools to create optimal settings for SLM formulations. The suggested optimized formulation was not only formulated but also evaluated (21-22).

\section{RESULTS AND DISCUSSION}

\section{Rheological properties}

Flow behavior of the prepared SLMs was analyzed and the outcomes of rheological analysis are given in Table I. There was no significant difference in flow properties between the SLM formulations; however, the wax-surfactant ratio influenced the rheology of SLMs. Higher surfactant concentration with lower lipid matrix concentration had a positive effect on the flow behavior of SLMs because such formulations had smoother and spherical shapes. The results of Carr's index for all SLMs ranged from 12 to 18, demonstrating a good flow character of SLMs. Outcomes obtained from the angle of repose remained lower than 25, which also corroborated the good flow character of SLMs. Similar findings observed from the results of Hausner's ratio confirmed the good rheological behavior of SLMs (19). It remained below 1.5 for all formulations.

\section{Data optimization and model validation}

A mathematical relationship was established between the independent factors $X_{1}(C W$ concentration), $X_{2}$ (GMS concentration) and $X_{3}$ (T-20 concentration) and responses $Y_{1}(P Y)$, $Y_{2}(E E), Y_{3}\left(D R_{12}\right)$ and polynomial equations representing the impacts of these factors alone and their interactions on responses were obtained. Independent factors were those varied to test and check changes in dependent variables (responses) and the effects on dependent variables were measured and recorded. The ranges of responses (Table I) $Y_{1}, Y_{2}$ and $Y_{3}$ were 29-86, 45-83 and 48-86\%, respectively, and the ratio of maximum to minimum for the studied responses $\left(Y_{1}=2.96, Y_{2}=1.84\right.$ and $\left.Y_{3}=1.79\right)$ was found to be lower than three. In such a case, no further transformation of the model is suggested and power transformation may have little effect. The applied CCRD design suggested that all of the studied responses followed a quadratic model. Significance of the quadratic model was analyzed with the aid of analysis of variance (ANOVA) applied at the $5 \%$ significance level and a $p$-value lower than 0.05 indicated that the model was significant. The selected model was further assessed for the lack of fit (LOF) and for the studied responses; LOF was not found to be significant (21-22). The values of $R^{2}$, adjusted $R^{2}$, predicted $R^{2}, \mathrm{SD}, \% \mathrm{CV}$ (Table II) and the analysis of variance results for each response (ANOVA) in Table II confirmed that the model was significant for the three studied responses. The predicted $R^{2}$ values for $Y_{1}, Y_{2}$ and $Y_{3}$ of $0.7896,0.7715$ and 0.7589 were found to be very close to adjusted $R^{2}$ values of $Y_{1}$ (0.8334) and $Y_{2}(0.8425)$ and $Y_{3}(0.8436)$, respectively (Table II). The signal to noise ratio was measured with adequate precision and its values for $Y_{1}(19.396), Y_{2}(13.634)$ and $Y_{3}(12.036)$ were found to be higher than 4 , indicating the adequacy and suitability of the model for the studied responses (23-24).

For every SLMs formulation, the results of percentage yield (Table I) showed significant variation from 29 (F9) to 86 \% (F20). Higher concentration of CW or GMS along with 
M. Hanif et al.: A Sustained release biodegradable solid lipid microparticles: Formulation, evaluation and statistical optimization by response surface methodology, Acta Pharm. 67 (2017) 441-461.

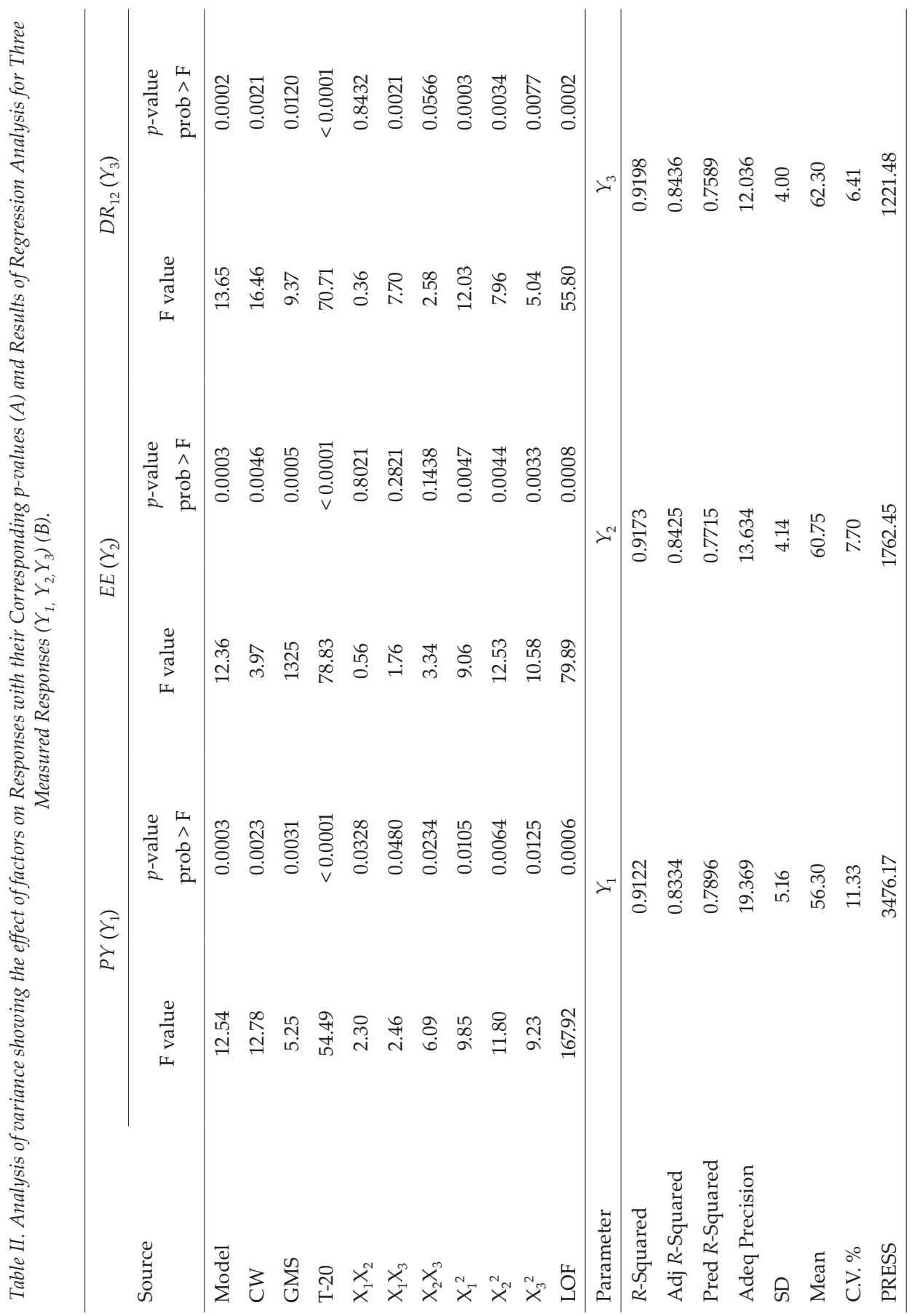


lower T-20 (F1, F8, F9, F12) showed lower than $50 \%$ percentage yield. Lower yield of SLMs may be associated with higher aggregation of wax because of poorer stabilization of lipid droplets at lower concentrations of T-20. Higher concentrations of the lipid matrix along with higher T-20 (F3, F16) concentration were found to be good formulation parameters, because $P Y$ higher than $75 \%$ was observed in these formulations (12). Similar findings were published in literature for biodegradable microparticulate systems (11-13). With a similar concentration of T-20, higher PY (53\%) was observed for the formulations containing higher CW concentrations (F12) compared to the formulations (F1; PY of $32 \%$ ) with higher GMS concentrations. The equation obtained for $Y_{1}$ with actual values is shown below:

$$
\begin{gathered}
Y_{1}=+133.56-52.33 X_{1}-75.39 X_{2}-47.73 X_{3}+9.28 X_{1} X_{2}+9.20 X_{1} X_{3}+18.80 X_{2} X_{3} \\
+14.36 X_{1}^{2}+15.61 X_{2}^{2}+21.65 X_{3}^{2}
\end{gathered}
$$

For $Y_{1}$, all the three factors alone had a negative impact on $P Y$, indicating that an increase in concentration of either lipids or surfactant could not increase the $P Y$. The F-value for $Y_{1}$ was found to be 12.54, indicating that the model was significant $(p<0.0001)$. Similarly, the "Prob $>F^{\prime \prime}$ at a level of less than 0.05 showed that model terms were also significant (24). For $Y_{1}$, the $p$-values of $X_{1}, X_{3}, X_{1} X_{3}$ were $<0.0001$, showing that the CW concentration, surfactant concentration and $\mathrm{CW}$ with surfactant were the most significant terms. This suggests that both lipids alone could not formulate SLMs in the absence of T-20. The interaction of lipids with T-20 was found to be synergistic, causing an increase in $P Y$ of SLMs. Three dimensional surface graphs indicating the interaction effect of two factors keeping the third factor at a constant level like the impact of $X_{1}-X_{3}$ on $Y_{1}$ are presented in Fig. 1a-c. The actual values of response $Y_{1}$ were found to be very close to the predicted values of $Y_{1}$.

Surfactant concentration played a vital role in the development and stabilization of droplets in emulsion, which finally contributed to the increase in PY of SLMs. At lower surfactant concentrations, the yield remained very low and increased lipid concentration with lower surfactant concentration caused more aggregation and accumulation of lipids instead of SLM production (2-3).

Responses $Y_{2}$ and $Y_{3}$ also followed a quadratic model and suggested regression equations for both responses are given below:

$$
\begin{aligned}
& Y_{2}=+89.76-20.45 X_{1}- 37.29 X_{2}-26.69 X_{3}-0.97 X_{1} X_{2}+6.00 X_{1} X_{3}+9.20 X_{2} X_{3} \\
&+ 7.09 X_{1}^{2}+11.17 X_{2}^{2}+16.03 X_{3}^{2} \\
& Y_{3}=+91.23-16.17 X_{1}-1.32 X_{2}-19.132_{3}-0.65 X_{1} X_{2}-3.20 X_{1} X_{3}-6.40 X_{2} X_{3} \\
&+6.47 X_{1}^{2}+6.45 X_{2}^{2}+10.09 X_{3}^{2}
\end{aligned}
$$

The application of ANOVA to statistically validate the polynomial equations and the model for $Y_{2}$ and $Y_{3}$ suggested a level of significance $(p)$ less than 0.05 , indicating that these equations could be applied to forecasting the effects of all variables on the EE and drug 
a)

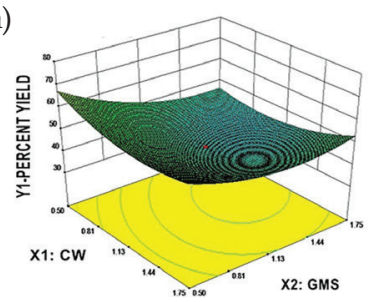

d)

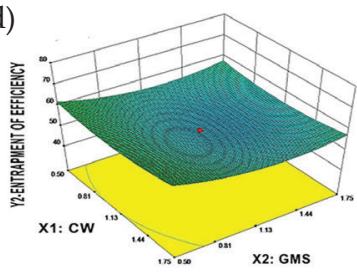

g)

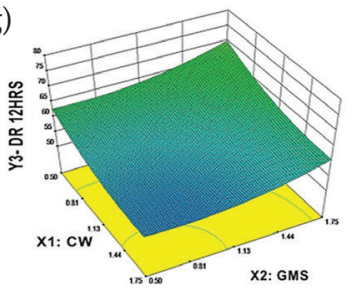

b)

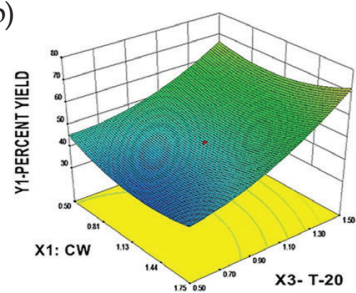

e)

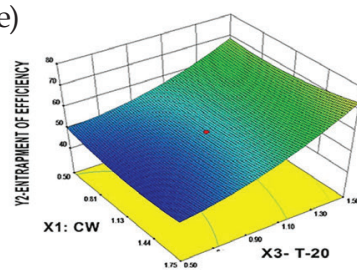

h)

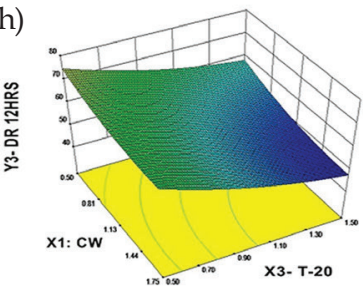

c)

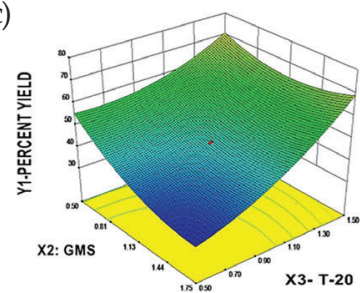

f)

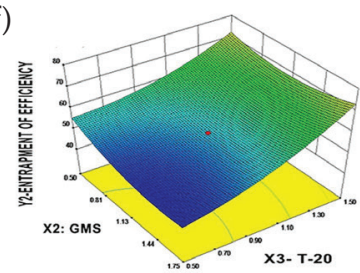

i)

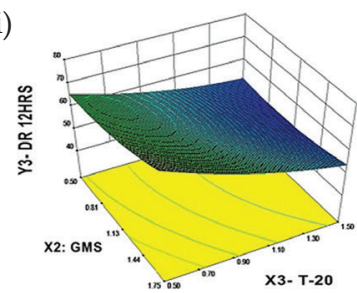

Fig. 1. 3D response surface plots showing the effect of carnauba wax $\left(X_{1}\right)$, glyceryl monostearate concentrations $\left(X_{2}\right)$ and surfactant concentrations $\left(X_{3}\right)$ alone and in combination on percent yield $\left(Y_{1}\right)$ $(\mathrm{a}-\mathrm{c})$, on entrapment efficiency $\left(Y_{2}\right)(\mathrm{d}-\mathrm{f})$ and on drug release at $12 \mathrm{~h}\left(Y_{3}\right)(\mathrm{g}$-i).

release at $12 \mathrm{~h}$. The F-values for $Y_{2}$ and $Y_{3}$ were found to be 12.36 and 13.65, respectively, indicating that the model was significant $(p<0.0001)$. It can be concluded from the regression equation that the $E E$ and drug release from prepared SLMs were strongly dependent on the studied variables $(19,22)$.

Assessment of $E E$ for every SLM formulation showed great variation, from 45 (F1, F10) to $83 \%$ (F19) (Table I). The percent drug loading (DL) varied from 14 (F1) to $45 \%$ (F16). The $E E$ and $D L$ increased with an increase in lipid matrix concentration providing an equal increase in T-20 concentration as observed for F2, F3, F10 and F16. Formulations F3 and F9 had equal concentrations of CW and GMS but both formulations had different $E E$ and $D L$ because of the T-20 concentration difference applied in the preparation of these formulations (26). Formulations with lower percentage of CW or GMS at lower concentration of T-20 (F1, F12) showed minimum EE. Higher concentration of CW or GMS could not increase EE as observed for F8, F9 (46 and $45 \%$ ) because a small amount of T-20 (0.16 and 0.50 $\%)$ was available to solidify lipid droplets for the entrapment of NEB. Thus, increase in $E E$ and $D L$ depended on both the enhancement of CW/GMS concentration and surfactant T-20 concentration. It is suggested that higher concentration of T-20 not only prevented a loss of drug in the external phase but was also responsible for the stabilization of lipid microparticles in the external phase. 
Significant terms observed for $Y_{2}$ and $Y_{3}$ were $X_{1}, X_{2}, X_{3}, X_{1}^{2}, X_{3}{ }^{2}$, indicating their potential impact on $E E$ and drug release. For $Y_{2}, X_{1} X_{2}, X_{1}^{2}, X_{3}^{2}$, and $X_{2}^{2}$ had a positive impact on $E E$, indicating that increase in CW and GMS concentration caused an increase in $E E$ if there was an equal increase in T-20 concentration. Moreover, combination of lipids also had a negative impact on $Y_{2}(E E)$, which might be due to the inability of T-20 concentration to emulsify and solidify SLMs droplets. Interaction of CW and GMS with T-20 was found to be positive, indicating a synergistic impact of T-20 on lipids to enhance EE.

Owing to their insoluble nature, lipids remain optimal materials for designing controlled release drug delivery systems. Drug release graphs (Fig. 2) showed that the time of NEB release was strongly associated with lipid concentration (2). For $Y_{3}$, lower concentration of all three variables had a negative impact on drug release while higher concentration of these variables had a positive influence on drug release. It was thus concluded that an increase in CW/GMS concentration could successfully retard drug release over 12 hours. The equation suggested that the effect of $X_{1}(C W)$ in decreasing the release rate was found to be better compared to $X_{2}$ (GMS). For a similar concentration of T-20, the formulation with higher CW concentration (F12) showed more sustained and controlled drug release compared to formulations containing higher GMS concentrations (F1). The above fact could be associated with higher molecular weight and the more hydrophobic character of CW compared to GM, which caused greater reduction in medium diffusion inside SLMs. Similarly, the interaction terms $\left(X_{1} X_{3}\right)$ and $\left(X_{2} X_{3}\right)$ were found to be negative, suggesting that the interaction of CW and GMS with T-20 was useful in decreasing/controlling the NEB release. Moreover, drug release studies at the gastric $\mathrm{pH}$ of 1.2 showed less than $10 \%$ of NEB release, suggesting a gastro protective effect of lipids due to their insoluble nature, as also mentioned in the literature (26).

SLMs formed at lower surfactant concentration released the drug faster compared to SLMs formed at higher surfactant concentration (4). This may be due to the fact that SLMs
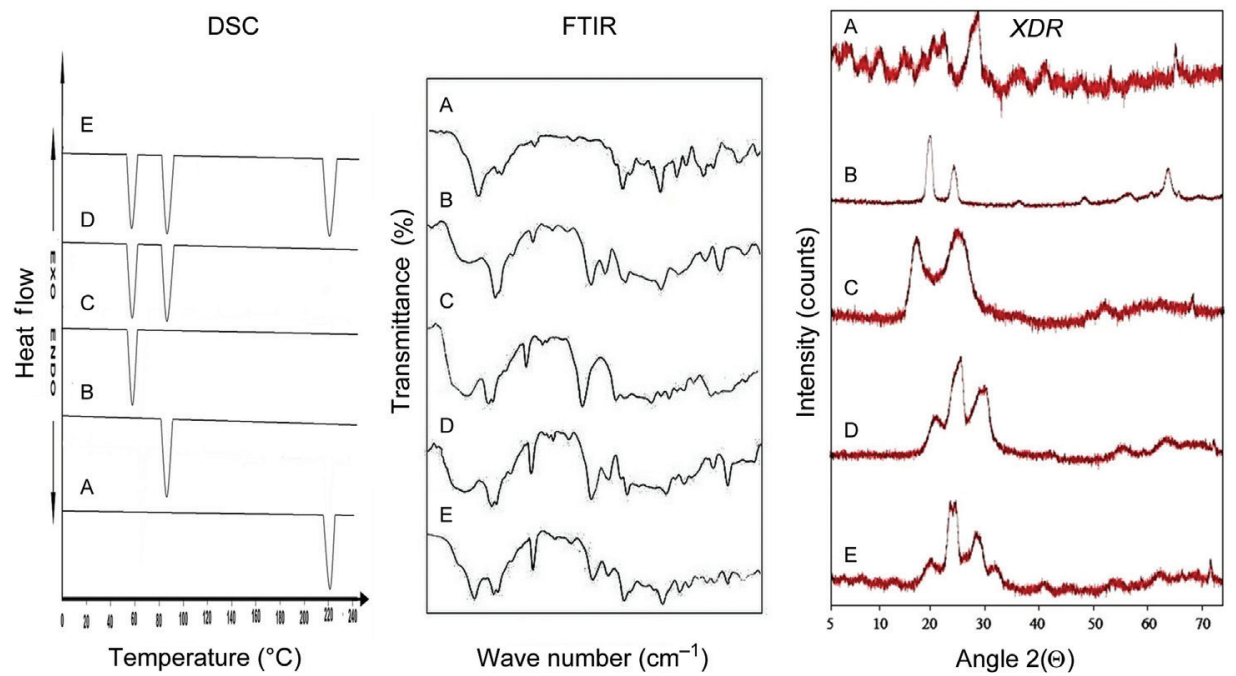

Fig. 2. Cumulative drug release profiles (\%) of F1-F5 (A), F5-F10 (B), F11-F15 (C) and F16-F20 (D) at pH 6.8. 


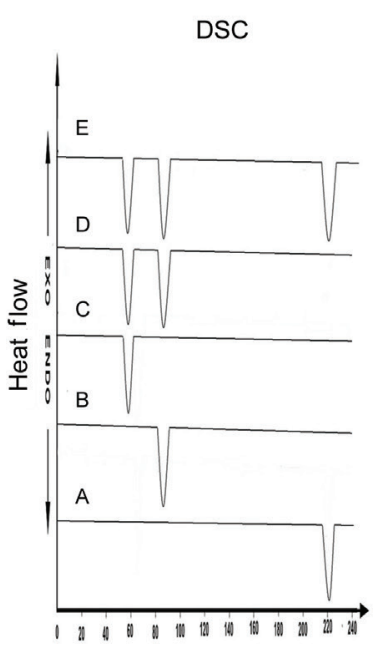

Temperature $\left({ }^{\circ} \mathrm{C}\right)$
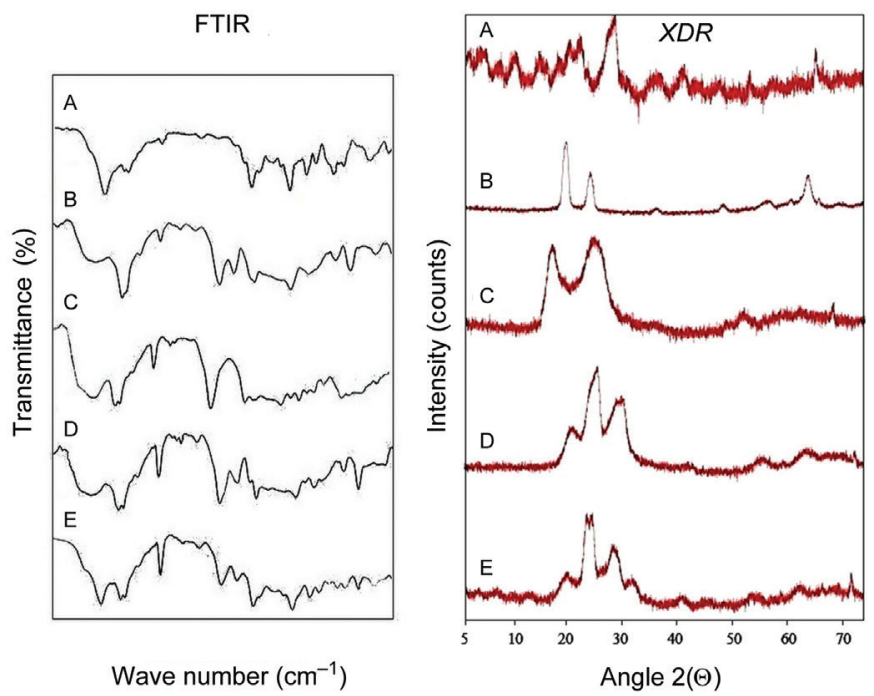

Fig. 3. DSC thermograms of nebivolol (A), carnauba wax (B), glyceryl monostearate (C), mixture of carnauba wax and glyceryl monostearate (D), optimized formulation of nebivolol-loaded SLMs (E). FTIR spectra of nebivolol (A), carnauba wax (B), glyceryl monostearate (C), mixture of carnauba wax and glyceryl monostearate (D), optimized formulation of nebivolol-loaded SLMs (E) and XRD Patterns of nebivolol (A), carnauba wax (B), glyceryl monostearate (C), blank SLMs (D) optimized formulation of nebivolol-loaded SLMs (E).

formed at lower surfactant concentration could have cracks and holes on the surface causing faster drug release like in the case of formulation F8 (drug release at $12 \mathrm{~h} 86 \%$; Fig. 4b). Higher concentration of lipids induces a higher hydrophobic character and decreases water diffusion in the lipid matrix, leading towards a slower drug release rate (1). Formulation F19 showed very slow drug release rates because of the presence of higher lipid (CW) con-
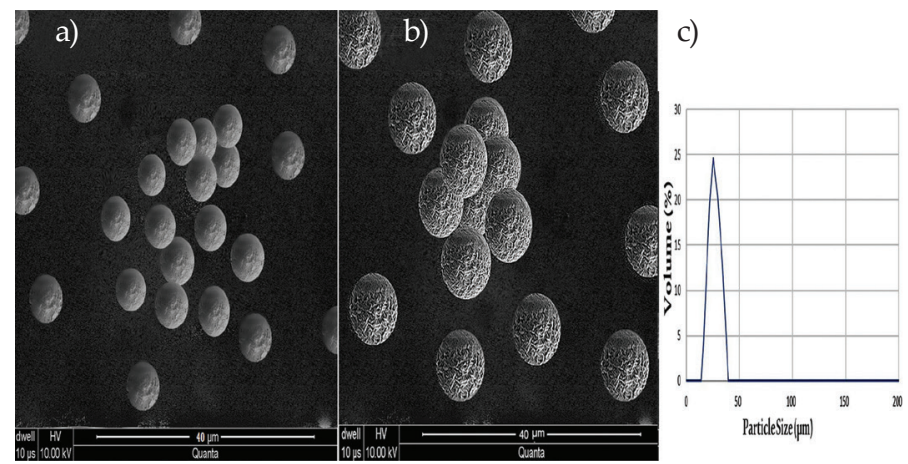

d)

Fig. 4. SEM photomicrographs of the: a) optimized formulation, b) SEM image of formulation F8, c) particle size distribution curve and d) zeta-potential distribution curve of the optimized formulation. 
centration. Interaction effects of CW concentration $\left(X_{1}\right)$ and GMS $\left(X_{2}\right)$ on $Y_{2}$ and $Y_{3}$ are shown in Fig. 1d-i.

Impacts of CW, GMS and T-20 concentration were found to be significant for all of the studied responses and the impact of $\mathrm{CW}$ and T-20 could be considered more prominent compared to that of GMS on the studied responses. In polynomial equations, the coefficients for interaction terms (combination of variables) were found to be positive, indicating a synergistic effect of variables on responses. For response $Y_{3}$, it was objective to decrease the drug release over 12 hours. It was observed that $\mathrm{CW}$ had a more prominent effect than that of GMS. It was also observed that the connection between variables and observed responses was not linear. Furthermore, a change of more than one variable at the same time at different levels produced quite a different intensity of the response $(24,26)$.

The numerical optimization technique was applied to set optimum conditions of the process variables $\left(X_{1}, X_{2}, X_{3}\right)$ for attaining desirable results for responses $Y_{1}, Y_{2}$ and $Y_{3}$ of SLM formulations. Desirability of the studied responses was calculated on a scale of $0-1$ with the aid of software and was found to be close to one (17). Selection of optimum SLM formulations was made on the basis of achieving maximum PY $(90 \%)$, maximum EE (85\%) but minimum drug release at $12 \mathrm{~h}$ (less than $50 \%$ ), since controlling the drug release rate remains a critical factor in designing sustained release drug delivery systems. The optimized formulation OF1 (Table III) suggested by software was prepared and characterized for the responses. Comparison of the predicted and observed values of three responses for the optimized formulation was made and the calculated prediction error (equation 18) was found to be very low, as shown in Table III. It was quite obvious that polynomial equations sufficiently addressed the impact of independent formulation variables on the studied responses, suggesting adequateness and suitability of optimization techniques by CCRD for optimizing the SLM formulation (24-25).

$$
\text { PE }(\%)=\text { Observed value }- \text { Predicted value } / \text { Predicted value } \times 100
$$

\section{Drug release kinetics}

Different drug release kinetic models such as zero order, first order, Korsmeyer-Peppas, Hixson Crowell and Higuchi models were applied to the analyzed drug release profiles of prepared SLM formulations and the outcomes for release constants and regression coefficients are shown in Table IV. Drug release profiles dominantly followed zero order

Table III. The suggested optimized formulation composition, predicted and observed levels of responses, prediction errors and desirability level

\begin{tabular}{lcccccc}
\hline $\begin{array}{l}\text { Independent } \\
\text { variable }\end{array}$ & $\begin{array}{c}\text { Optimum } \\
\text { level }\end{array}$ & $\begin{array}{c}\text { Dependent } \\
\text { variable/ } \\
\text { response }\end{array}$ & $\begin{array}{c}\text { Predicted } \\
\text { value }\end{array}$ & $\begin{array}{c}\text { Observed } \\
\text { value }\end{array}$ & $\begin{array}{c}\text { Prediction } \\
\text { errors }\end{array}$ & $\begin{array}{c}\text { Desirability } \\
\text { level }\end{array}$ \\
\hline$X_{1}$ & 1.75 & $Y_{1}$ & 80.86 & 75.8 & 1.04 & \\
$X_{2}$ & 1.75 & $Y_{2}$ & 77.64 & 78.8 & 1.5 & 0.889 \\
$X_{3}$ & 1.50 & $Y_{3}$ & 51.08 & 46.7 & 1.4 & \\
\hline
\end{tabular}


and Higuchi models because the obtained $R^{2}$ values for these models were found to be higher compared to the values of $R^{2}$ for the other models applied. According to the zero order model, the NEB release from SLMs was independent of the remaining drug concentration in SLMs while the Higuchi model suggests the diffusion mechanism of drug release. The $n$ value ranged from 0.87 to 1.07 (anomalous mechanism of drug release), suggesting that drug release was not only controlled by diffusion but also erosion of biopolymers. Thus the likely mechanism for release of encapsulated NEB from SLMs would be drug diffusion from the hydrophobic lipid matrix of SLMs (20).

\section{Fourier transform infrared spectroscopy (FTIR)}

FTIR study demonstrated good compatibility of NEB with both encapsulating agents (CW and GMS). Individual spectra of NEB, GMS and CW were compared with the FTIR spectrum of the prepared drug loaded SLMs, as shown in Fig. 3 (Section FTIR). Characteristic aliphatic $\mathrm{N}-\mathrm{H}$, alkenes $\mathrm{C}=\mathrm{C}$ and $\mathrm{C}-\mathrm{H}$ stretches were observed at 3185, 2319 and 1490 $\mathrm{cm}^{-1}$, respectively, not only in the NEB spectrum but also in NEB-loaded OF1. Similarly, carbonyl and CO-ester groups were also visible at 1536 and $1074 \mathrm{~cm}^{-1}$, respectively, in the FTIR-spectrum of NEB-loaded SLMs.

The spectra of NEB alone and of NEB-loaded SLMs also indicated major stretches for aliphatic $\mathrm{CH}$ groups and alcoholic and phenolic $\mathrm{OH}$ groups at 2870 and $3650 \mathrm{~cm}^{-1}$, respectively. FTIR spectra did not show the absence or shift of any principal peaks of NEB or CW or GMS either in the FTIR spectrum of NEB-loaded SLMs or in individual FTIR spectra of NEB, CW and GMS. These findings confirmed the compatibility of NEB and lipids used for the preparation of SLMs (26).

\section{Differential scanning calorimetry (DSC)}

Compatibility of NEB with CW and GMS was analyzed with the help of differential scanning calorimetry. DSC thermograms of NEB, CW and drug loaded SLMs are presented in Fig. 3 (Section DSC). Specific endothermic peaks relevant to melting points of CW and GMS were quite visible at a temperature of $85^{\circ} \mathrm{C}$ for $\mathrm{CW}$ alone (B) and at $59^{\circ} \mathrm{C}$ for GMS (C), as shown in Fig. 3 (Section DSC). The presence of a particular peak associated with the melting of NEB in drug loaded SLMs at a temperature of $221^{\circ} \mathrm{C}$ was also confirmed. However, a decrease in peak intensity and lowering of the NEB melting peak temperature indicated that some fraction of NEB still existed in crystalline form in the prepared SLMs (E). Similarly, in NEB-loaded SLMs, particular peaks corresponding to CW and GMS melting points were also visible at temperatures of 85 and $59^{\circ} \mathrm{C}$, respectively, showing compatibility of the lipids and the drug $(4,26)$. All of these observations clearly support the fact of NEB inclusion in the prepared SLMs.

\section{X-ray power diffraction studies}

X-ray power diffraction studies of optimized formulations were performed and the XRD patterns of individual drugs (3A of section XRD), individual lipids (3B and 3C of section XRD), blank SLMs (3D of section XRD) and prepared NEB-loaded optimized SLMs (3E of section XRD) were compared for any addition or deletion of typical components (Fig. 3 
XRD). The comparison indicated the presence of characteristic NEB peaks (Fig. 3E) without any impact on the diffraction position. However, a reduction in the intensity of NEB peaks indicated that some of the drug was still in crystalline form in SLMs. The XRD examination of NEB-loaded SLMs (Fig. 3E of section XRD) revealed sharp as well as scattered peaks, proving that a fraction of NEB was changed into amorphous form while formulating SLMs. This change in crystallinity may also cause drug expulsion and may alter the drug release rate (28). The peaks for NEB were observed at $2 \theta$ of $20^{\circ}, 25^{\circ}$ and $30^{\circ}$, indicating the crystalline nature of NEB (Fig. 3A). There was complete absence of any prominent alteration in the diffraction position relevant to NEB, suggesting that formulation steps of NEB-loaded SLMs did not produce any unfavorable effects on NEB (7). Moreover, the smoothness of lipid peaks in Fig. 3D and 3E indicated formation of NEB-loaded SLMs.

\section{Scanning electron microscopy (SEM)}

The prepared SLMs of the optimized formulation (OF1) were found to be spherical in shape, as shown in Fig. 4a. Most of the studies in literature have also reported the spherical shape of solid lipid microparticles (27). The surface of SLMs made with higher T-20 concentrations was found to be smooth because at higher level of T-20, lipid droplets could solidify better and yield spherical smooth surfaced SLMs (19). At the same time, holes in the surface of SLMs of OF1 made with higher lipid concentration were quite invisible (2, 5). The absence of these holes facilitated controlled release of NEB from SLMs. The SEM image (Fig. $4 \mathrm{~b}$ ) of formulation (F8) with maximum drug release ( $86 \%$ ) clearly indicated the presence of holes and a rough surface, which may be attributed to minimum concentration of T-20 that could not emulsify and solidify lipid droplets properly. SEM studies also indicated that the optimized SLMs had an average size of $40 \mu \mathrm{m}$.

\section{Zeta-potential and particle size measurements}

The particle size distribution and zeta-potential of the optimized formulation (OF1) were determined with the help of the electrophoretic light scattering method (Fig. 4c and $4 \mathrm{~d}$, respectively). Size distribution of SLMs ranged from 10 to $40 \mu \mathrm{m}$ while the major fraction of SLMs had an average size of $25 \mu \mathrm{m}$. Particle size was greatly influenced by the concentration of lipids and concentration of T-20 (28). Formulation OF1 showing smaller size and a narrow size distribution because T-20 at a concentration level of $1.75 \% \mathrm{~m} / \mathrm{V}$ greatly contributed to the yield of micron size SLMs as SLM preparation without surfactant during the preliminary stage failed. Higher T-20 concentration played a role in preventing the aggregation of hydrophobic lipid microparticles (19-20).

Stabilty of SLMs was deduced from the measurement of zeta-potential, which is the electrical/charge potential at the shear plane. Particles with higher zeta-potential would prevent microparticle aggregation due to higher interparticle repulsion and could be regarded as having better storage stability. In the current study, the zeta-potential of OF1 was observed in the range of -20 to $-40 \mathrm{mV}$ (Fig. 4c), indicating that SLMs should have better stability. Minimum value of $30 \mathrm{mV}$ is usually necessory for better stability of SLMs (28). Similar findings from Mishara et al. (2009) are reported in literature (28). The presence of high intensity of negative charge would generate electrostatic repulsion between SLMs, disallowing aggregation of SLMs (25). The negative zeta-potential value of optimized 
M. Hanif et al.: A Sustained release biodegradable solid lipid microparticles: Formulation, evaluation and statistical optimization by response surface methodology, Acta Pharm. 67 (2017) 441-461.

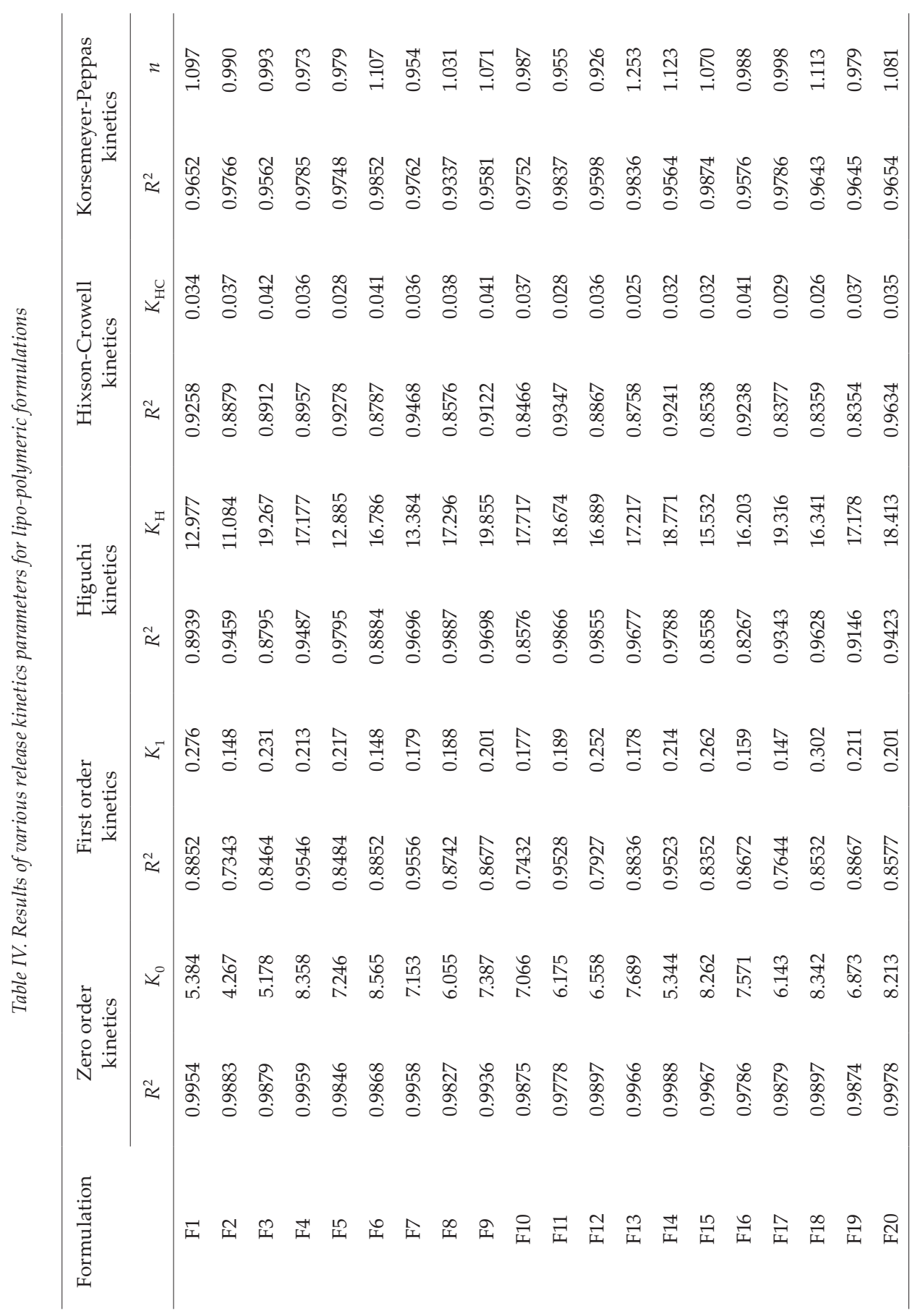


SLMs was expected because formulation ingredients like CW consist of aliphatic esters, ester of 4-hydroxycinnamic acid, hydroxycarboxylic acid, fatty alcohols and GMS consists of glycerol esters of fatty acid. T-20 is a type of non-ionic surfactant and is considered neutral and not able to increase the zeta-potential values. Such results were obtained for NLC prepared with the same wax and surfactant but loaded with benzophenone-3 (29). The presence of free fatty acids in lipids and emulsifier such as T-20 may contribute negative charge to SLMs because the manufacturing of T-20 and other surfactants involves free fatty acids as substrate (29).

\section{CONCLUSIONS}

NEB loaded SLMs were successfully formulated by the solvent evaporation method and then characterized for rheological behavior, zeta size, zeta potential and morphology. With the help of a central composite rotatable design (CCRD), concentration levels of the selected formulation parameters such as CW concentration $(1.75 \%, \mathrm{~m} / \mathrm{m})$, GMS concentration $(1.75 \%, \mathrm{~m} / \mathrm{m})$ and surfactant concentration $(1.50 \%, \mathrm{~m} / \mathrm{m})$ were optimized with the intention to achieve minimum drug release and maximum percentage yield and entrapment efficiency. The optimized SLM formulation (OF1) showed $46 \%$ drug release after $12 \mathrm{~h}, 75$ $\%$ percentage yield and $78 \%$ entrapment efficiency. Smooth, spherical, free flowing SLMs with a size range of 10 to $40 \mu \mathrm{m}$ and a zeta-potential of -20 to $-40 \mathrm{mV}$ were formulated. FTIR and DSC analysis confirmed compatibility of the drug with lipids. Powder X-RD studies showed that the drug retained its crystalline form during the encapsulation process. Drug release kinetics indicated the Higuchi drug release model with a possible mechanism of erosion and diffusion, as shown from the value of $n>0.85$. CW and GMS proved to be suitable release retarding materials. CW was found to be a better carrier than GMS for achieving desired responses in the prepared sustained release SLMs but the role of T-20 was found to be a much more critical factor compared to the other two variables. Furthermore, sustained release SLMs loaded with NEB could offer possible benefits of controlling the heart rate for 24 hours in patients with chronic hypertension and could be helpful in minimizing side effects and treatment costs.

\section{REFERENCES}

1. J. M. Flack and S. A. Nasser, Benefits of once-daily therapies in the treatment of hypertension, Vasc. Health Risk Manag. 7 (2011) 777-787; https://doi.org/10.2147/VHRM.S17207

2. R. Bodmeier, J. Wang and H. Bhagwatwar, Process and formulation variables in the preparation of wax microparticles by melt dispersion technique for water insoluble drugs, J. Microencapsul. 9 (1992) 89-98; https://doi.org/103109/02652049209021226

3. R. B. Oliveira, T. L. Nascimento and E. M. Lima, Design and characterization of sustained release ketoprofen entrapped carnauba wax microparticles, Drug Dev. Ind. Pharm. 38 (2012) 1-11; https:// doi.org/10.3109/03639045.2011.587433

4. C. M. Adeyeye and J. C. Price, Development and evaluation of sustained release ibuprofen-wax microspheres. I. Effect of formulation variables on physical characteristics, Pharm. Res. 8 (1991) 1377-1383; https://doi.org/10.1023/A:1015845022112

5. Y. A. Goma, I. A. Darwish, N. A. Boraei and L. K. El-Khordagui, Formulation of wax oxybenzone microparticles using a factorial approach, J. Microencapsul. 27 (2010) 628-639; https://doi.org/10.31 09/02652048.2010.506580 
6. L. M. Van Bortel, F. Fici and F. Mascagni, Efficacy and tolerability of nebivolol compared with other antihypertensive drugs: a meta-analysis, Am. J. Cardiovasc. Drugs 8 (2008) 35-44; https://doi. org/10.2165/00129784-200808010-00005

7. L. A. D. Silva, F. V. Teixeira, R. C. Sepra, N. L. Esteves, R. R. Dos-Santos, E. M. Lima, et al., Evaluation of carvedilol compatibility with lipid excipients for the development of lipid-based drug delivery systems, J. Therm. Anal. Calorim. 123 (2016) 2337-2344; https://doi.org/10.1007/ s10973-015-5022-1

8. K. Westesen and B. Siekmann, Solid lipid particles, particles of bioactive agents and methods for the manufacture and use thereof, U.S. Pat. 08/226, 471, 12 April 1994; Publication date July 28 (1998) 5785976 A.

9. S. N. Patere, N. S. Desai, A. S. Jain, P. P. Kadam, U. M. Thatta, N. Gogtay, C. J. Kapadia, N. Farah and M. S. Nagarsenker, Compritol ${ }^{\circledR} 888$ ATO, a lipid excipient for sustained release of highly water soluble active formulation, scale-up and IVIVC study, Curr. Drug Deliv. 10 (2013) 548-556; https://doi.org/10.2174/1567201811310050006

10. M. K. Shah, P. Madan and S. Lin, Preparation, in vitro evaluation and statistical optimization of carvedilol loaded solid lipid nanoparticles for lymphatic absorption via oral administration, Pharm. Dev. Technol. 19 (2014) 475-485; https://doi.org/10.3109/10837450.2013.795169

11. M. R. Priya and N. Jeevitha, semi-solid dispersion of carvedilol solid lipid nanoparticles for topical delivery, EJPMR 3 (2016) 231-238.

12. H. M. Aboud, M. H. El Komy, A. A. Ali, S. F. El Menshawe and A. Abdelbary, Development, optimization, and evaluation of carvedilol-loaded solid lipid nanoparticles for intranasal drug delivery, AAPS PharmSciTech 17 (2016) 1353-1365; https://doi.org/10.1208/s12249-015-0440-8

13. A. A. Attama, S. Reichl and C. C. Muller-Goymann, Sustained release and permeation of timolol from surface-modified solid lipid nanoparticles through bioengineered human cornea, Curr. Eye Res. 34 (2009) 698-705; https://doi.org/10.1080/02713680903017500

14. S. N. Patere, C. J. Kapadia and M. S. Nagarsenker, Influence of formulation factors and compression force on release profile of sustained release Metoprolol tablets using Compritol ${ }^{\circledR} 888$ ATO as lipid excipient, Indian J. Pharm. Sci. 77 (2015) 620-625; https://doi.org/10.4103/0250-474X.169030

15. A. Bruzzo, T. Cerchiara, F. Bigucci, M. C. Gallucci and B. Luppi, Mucoadhesive buccal tablets based on chitosan/gelatin microparticles for delivery of propranolol hydrochloride, J. Pharm. Sci. 104 (2015) 4365-4372; https://doi.org/10.1002/jps.24688

16. S. S. Sule and W. Frishman, Nebivolol: new therapy update, Cardiol Rev. 14 (2006) 259-264; https://doi.org/10.1097/01.crd.0000223651.03023.8e

17. W. Chaisri, W. E. Hennink, C. Ampasavate and S. Okonogi, Cephalexin microspheres for dairy mastitis: Effect of preparation method and surfactant type on physicochemical properties of the microspheres, AAPS PharmSciTech. 11 (2010) 945-951; https://doi.org/10.1208/s12249-0109453-5

18. X. Wu, G. Li and Y. Gao, Optimization of the preparation of nalmefene-loaded sustained-release microspheres using central composite design, Chem. Pharm. Bull. 54 (2006) 977-981; https://doi. org/10.1248/cpb.54.977

19. S. Milak, N. Medlicott and I. G. Tucker, Solid lipid microparticles containing loratadine prepared using a micromixer, J. Microencapsul. 23 (2006) 823-831; https://doi.org/10.1080/09687860600945750

20. B. Albertini, N. Passerini, M. L. Gonzalez-Rodriguez, B. Perissutti and L. Rodriguez, Effect of Aerosil on the properties of lipid controlled release microparticles, J. Control. Release 100 (2004) 233-246; https://doi.org/10.1016/j.jconrel.2004.08.013

21. S. Mukherjee, S. Ray and R. S. Thakur, Solid lipid nanoparticles: a modern formulation approach in drug delivery system, Indian J. Pharm. Sci. 71 (2009) 349-358; https://doi.org/10.4103/0250474X.57282 
22. J. Hao, X. Fang, Y. Zhou, J. Wang, F. Guo, F. Li and X. Peng, Development and optimization of solid lipid nanoparticle formulation for ophthalmic delivery of chloramphenicol using a BoxBehnken design, Int. J. Nanomedicine. 6 (2011) 683-692; https://doi.org/10.2147/IJN.S17386

23. C. Narendra, M. S. Srinath and R. Prakash, Development of a three layered buccal compact containing metoprolol tartrate by statistical optimization technique, Int. J. Pharm. 304 (2005) 102-114; https://doi.org/10.1016/j.ijpharm.2005.07.021

24. B. C. Nandy and B. Mazumder, Formulation and characterizations of delayed release multi-particulates system of indomethacin: optimization by response surface methodology, Curr. Drug Deliv. 10 (2014) 72-86; https://doi.org/10.2174/15672018109990041

25. D. V. Gowda and H. G. Shivakumar, Preparation and evaluation of waxes/fat microspheres loaded with lithium carbonate for controlled release, Indian J. Pharm. Sci. 69 (2007) 251-256; https://doi. org/10.4103/0250-474X.33152

26. S. B. Patil and K. K. Sawant, Development, optimization and in vitro evaluation of alginate mucoadhesive microspheres of carvedilol for nasal delivery, J. Microencapsul. 26 (2009) 432-443; https:// doi.org/10.1080/02652040802456726

27. L. Capretto, S. Mazzitelli and C. Nastruzzi, Design, production and optimization of solid lipid microparticles (SLM) by a coaxial microfluidic device, J. Control. Release 160 (2012) 409-417; https:// doi.org/10.1016/j.jconrel.2012.04.019

28. D. Jain and R. Banerjee, Comparison of ciprofloxacin hydrochloride-loaded protein, lipid and chitosan nanoparticles for drug delivery, J. Biomed. Mater. Res. B Appl. Biomater. 86 (2008) 105-112; https://doi.org/10.1002/jbm.b.30994

29. S. Lacerda, N. Cerize and M. Re, Preparation and characterization of carnauba waxnanostructured lipid carriers containing benzophenone-3, Int. J. Cosmet. Sci. 33 (2011) 312-321; https://doi. org/10.1111/j.1468-2494.2010.00626.x 